\title{
The inner jet of an active galactic nucleus as revealed by a radio-to- $\gamma$-ray outburst
}

\author{
Alan P. Marscher ${ }^{1}$, Svetlana G. Jorstad ${ }^{1,2}$, Francesca D. D'Arcangelo ${ }^{1}$, Paul S. Smith ${ }^{3}$, G. Grant Williams ${ }^{4}$, \\ Valeri M. Larionov ${ }^{2}$, Haruki Oh ${ }^{1} \uparrow$, Alice R. Olmstead ${ }^{1}$, Margo F. Aller ${ }^{5}$, Hugh D. Aller ${ }^{5}$, Ian M. McHardy ${ }^{6}$, \\ Anne Lähteenmäki ${ }^{7}$, Merja Tornikoski ${ }^{7}$, Esko Valtaoja ${ }^{8,9}$, Vladimir A. Hagen-Thorn ${ }^{2}$, Eugenia N. Kopatskaya ${ }^{2}$, \\ Walter K. Gear ${ }^{10}$, Gino Tosti ${ }^{11}$, Omar Kurtanidze ${ }^{12}$, Maria Nikolashvili ${ }^{12}$, Lorand Sigua ${ }^{12}$, H. Richard Miller ${ }^{13}$ \\ \& Wesley T. Ryle ${ }^{13}$
}

Blazars are the most extreme active galactic nuclei. They possess oppositely directed plasma jets emanating at near light speeds from accreting supermassive black holes. According to theoretical models, such jets are propelled by magnetic fields twisted by differential rotation of the black hole's accretion disk or inertialframe-dragging ergosphere ${ }^{1-3}$. The flow velocity increases outward along the jet in an acceleration and collimation zone containing a coiled magnetic field ${ }^{4,5}$. Detailed observations of outbursts of electromagnetic radiation, for which blazars are famous, can potentially probe the zone. It has hitherto not been possible to either specify the location of the outbursts or verify the general picture of jet formation. Here we report sequences of high-resolution radio images and optical polarization measurements of the blazar BL Lacertae. The data reveal a bright feature in the jet that causes a double flare of radiation from optical frequencies to $\mathrm{TeV} \gamma$-ray energies, as well as a delayed outburst at radio wavelengths. We conclude that the event starts in a region with a helical magnetic field that we identify with the acceleration and collimation zone predicted by the theories. The feature brightens again when it crosses a standing shock wave corresponding to the bright 'core' seen on the images.

The jet of BL Lac (Fig. 1) approaches us within $6-10^{\circ}$ of our line of sight at a flow speed of $0.981-0.994 c$ (where $c$ is the speed of light), corresponding to a Lorentz factor of $7.0 \pm 1.8$ (ref. 6). Relativistic aberration and the Doppler effect strongly beam the radiation, resulting in the apparent luminosity being hundreds of times higher than it would be if the emitting plasma were at rest. An essentially identical counterjet is presumably present, but too faint to detect because of

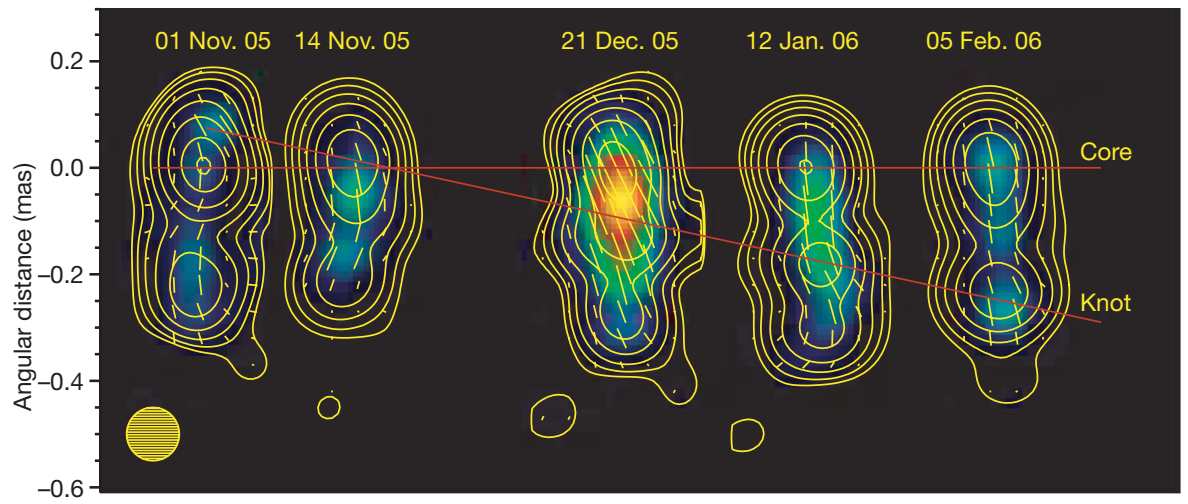

Figure $1 \mid$ Sequence of Very Long Baseline Array images of BL Lac at a wavelength of $7 \mathrm{~mm}$ (and a frequency of $43 \mathrm{GHz}$ ). The images are convolved with a circular gaussian function (shown in the bottom-left corner) that has a full width at half maximum of 0.1 mas, the resolution of the longest baselines of the array, which corresponds to $0.12 \mathrm{pc}$ at the distance of $291 \mathrm{Mpc}$ derived from the redshift, $z=0.069$, and the Hubble Law with an assumed slope of $H_{0}=72 \mathrm{~km} \mathrm{~s}^{-1} \mathrm{Mpc}^{-1}$ (ref. 22). The vertical axis shows angular distance relative to the core. The contours indicate total flux intensity, starting at 0.023 Janskys (Jy) per beam

$\left(1 \mathrm{Jy}=10^{-23} \mathrm{erg} \mathrm{s}^{-1} \mathrm{~cm}^{-2} \mathrm{~Hz}^{-1}\right)$ and increasing in factors of two. The colour scale represents polarized intensity, and peaks (yellow) at $0.215 \mathrm{Jy}$ per beam. The yellow line segments indicate the electric vector position angle (EVPA) of the polarization, which has an uncertainty of $\pm 7^{\circ}$ in each image. The EVPA has been rotated by $16^{\circ}$ in the clockwise direction to compensate for Faraday rotation; see the Supplementary Information. The approximate path of the centroid of the knot, the definition of which includes a point from an image obtained on 12 March 2006 in addition to those shown, is given by the slanted red line. The knot's observed proper motion of $1.2 \mathrm{mas} \mathrm{yr}^{-1}$ is equivalent to an apparent speed of $5.0 c$ after correction for time dilation due to cosmological redshift.

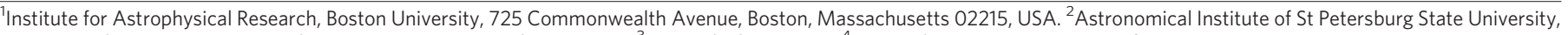
Universitetskij Prospect 28, Petrodvorets, 198504 St Petersburg, Russia. ${ }^{3}$ Steward Observatory, ${ }^{4}$ MMT Observatory, University of Arizona, Tucson, Arizona 85721-0065, USA. ${ }^{5}$ Astronomy Department, University of Michigan, 830 Dennison Building, Ann Arbor, Michigan 48109-1090, USA. ${ }^{6}$ Department of Physics and Astronomy, University of

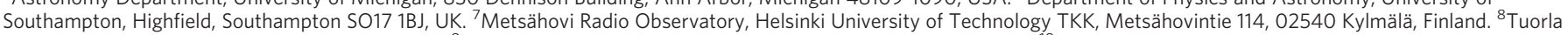

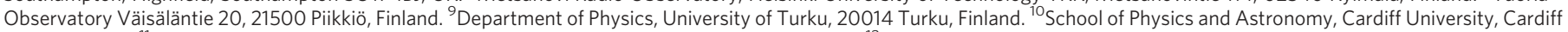

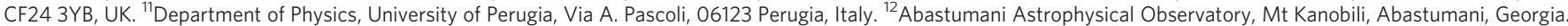
${ }^{13}$ Department of Physics and Astronomy, Georgia State University, Atlanta, Georgia 30303, USA. †Present address: Department of Physics, University of California, Berkeley, California 94720-7300, USA. 
beaming in the opposite direction. The stationary core lies at the northern end of the visible jet. Bright 'knots' emerge from the core at a rate of 1-2 per year and move south at apparent superluminal speeds, an illusion caused by their relativistic motion ${ }^{6}$.

The radio, optical, and X-ray light curves in Fig. 2 indicate a double flare in late 2005. The highly significant detection ${ }^{7}$ of $>0.2 \mathrm{TeV} \gamma$-rays from 2005.819 to 2005.831 during the first X-ray flare implies that acceleration of electrons with sub-TeV energies was particularly efficient at this time. These electrons can both produce $\mathrm{X}$-rays from synchrotron radiation and scatter the X-ray photons to $\mathrm{GeV} \gamma$-ray energies that are boosted to the $\mathrm{TeV}$ range by relativistic motion of the jet plasma. The location of such flares has been controversial: some observations ${ }^{8,9}$ indicate that they occur downstream of the core, whereas most theoretical models require that they take place well upstream of this region, where the plasma is more compact. As we explain below, our data indicate that the first flare in late 2005 corresponds to a disturbance passing through the zone upstream of the core, where the jet flow is still accelerating, and that the second occurs as the disturbance crosses a standing shock system in the core.

The identification of the location of the initial flare within the acceleration and collimation zone is significant, since previous observations of jet collimation are quite limited. For example, an image ${ }^{10}$ at 7-mm wavelength of the radio galaxy M87 appears to reveal an initially broad outflow that narrows into a nearly cylindrical jet. This is consistent with gradual collimation by either a toroidal magnetic field ${ }^{4}$ or external confining gas pressure that decreases with distance from the black hole ${ }^{11}$. The flow seen in M87 could include a 'sheath' that moves more slowly and is less focused than the 'spine' ${ }^{12}$. In the

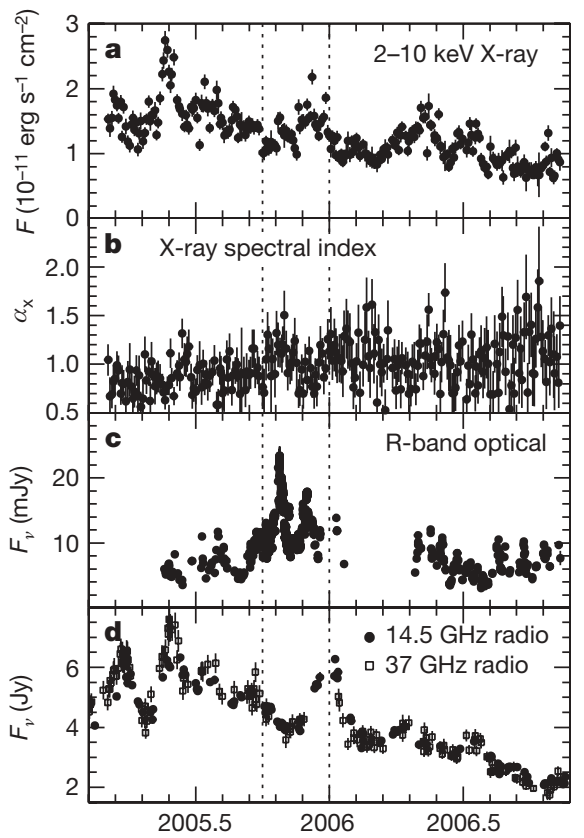

Figure 2 | Flux density at various wavebands and optical polarization of BL Lac, as functions of time. a-d, Dependence on time of the flux of radiation from BL Lac over a two-year interval at the indicated wavebands. The X-ray measurements in a are of photon energy flux $F$ integrated over photon energies of $2-10 \mathrm{keV}$. Error bars represent \pm 1 s.d. uncertainties in the values plotted. The exponent of the power-law dependence of X-ray flux density on frequency is denoted by $-\alpha_{\mathrm{x}}$. e-h, Enlargements of the 0.25 -yr time interval marked by vertical dotted lines in panels a-d, but with optical R-band EVPA $(\mathbf{g})$ and degree of polarization $P(\mathbf{h})$ respectively replacing X-ray spectral index (b) and radio flux density (d) (whereas e and $\mathbf{f}$ respectively show the magnified intervals in a and c). Error bars represent \pm 1 s.d. The interval of highly significant detections $s^{7}$ at photon energies $>0.2 \mathrm{TeV}$ is indicated by the width of the head of the arrow in e. The rotation in optical R-band EVPA near the time of the peak of the first optical and $\mathrm{X}$-ray flare is apparent. Because there is an ambiguity of $\pm 180^{\circ}$ in the value of the EVPA, we have selected the quadrant of each value that provides a consistent overall trend of case of BL Lac, the high apparent superluminal motions of bright knots in the jet and the pronounced variability at all wavelengths imply that the observed radiation arises exclusively from the spine, where special relativistic effects dominate.

The primary observational indicator of magnetic collimation requiring a coiled magnetic field in the spine of the jet is the evolution of the polarization. When observed at an angle to its axis, synchrotron radiation from a circularly symmetric jet with a helical field displays a net polarization oriented either parallel or perpendicular to the projected jet axis ${ }^{13}$. Such parallel and perpendicular polarizations can be confused with shock waves and velocity shear, respectively, which can produce the same polarization patterns. However, in a model where magnetic forces gradually accelerate and focus the jet, the flow velocity is directed along streamlines that follow a helical trajectory with a different, wider, pitch angle than that of the magnetic field ${ }^{5}$. The rotation of the flow can be traced back to the base of the jet in the orbiting accretion disk or differentially rotating ergosphere, where the spin of the black hole drags the inertial frames. A shock wave or other compressive feature propagating down the jet traces a spiral path that follows a streamline and cycles through the orientations of the helical field (see Fig. 3 and ref. 5). This should manifest itself as a rotation of the position angle of linear polarization as the feature moves outward. The degree of polarization should drop to a minimum in the middle of the rotation, when the mean magnetic field in the flaring region is transverse to that of the previously existing emission ${ }^{14}$. As Fig. $2 \mathrm{~g}$, h demonstrates, we see both effects.

The optical EVPA shown in Fig. $2 \mathrm{~g}$ rotates steadily by about $240^{\circ}$ over a five-day interval before settling at a value of $\sim 195^{\circ}$. The

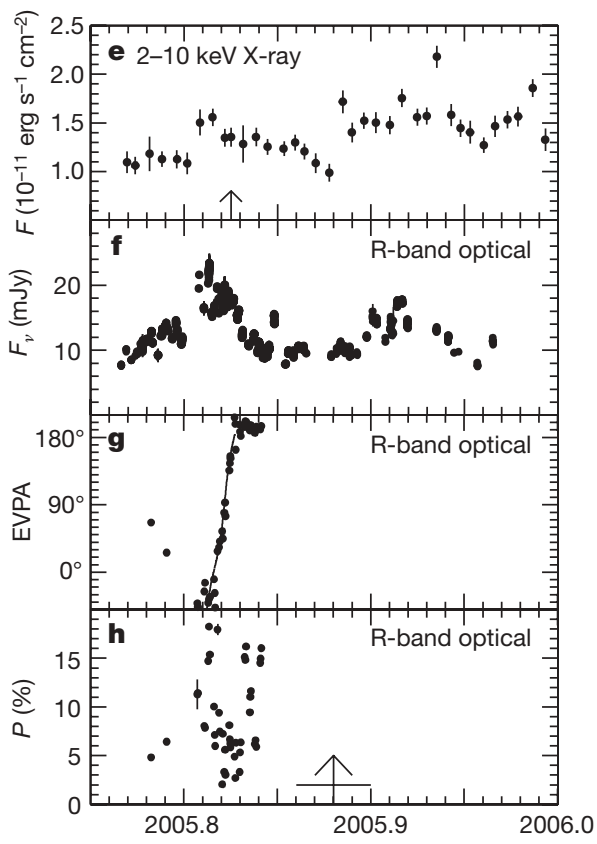

rotation between 2005.81 and 2005.83. The solid curve in $\mathbf{g}$ corresponds to the pattern predicted by the model shown in Fig. 3 when relativistic aberration is included. The vertical arrow (with error bar) in $\mathbf{h}$ indicates the time at which the superluminal knot is coincident with the stationary core seen in the images displayed in Fig. 1. Optical polarimetric data were obtained from Steward Observatory and the Crimean Astrophysical Observatory. Optical flux density points were obtained from photometry at these two sites plus Lowell Observatory, Perugia University Astronomical Observatory and the Abastumani Astrophysical Observatory. All of the optical telescopes are equipped with charge-coupled-device cameras. Measurements of X-ray flux and the continuum spectrum were obtained from a monitoring program with the NASA Rossi X-ray Timing Explorer. Measurements of radio flux density were obtained from the University of Michigan Radio Astronomy Observatory and the Metsähovi Radio Observatory. Descriptions of telescopes and data analysis are available in the Supplementary Information. 
sequence of images in Fig. 1 shows a bright, superluminal knot that first appears upstream of the core. It subsequently moves past the core and proceeds down the jet at a position angle of $\sim 190^{\circ}$ and with an EVPA that is parallel to the jet to within the observational uncertainty. The close correspondence between the optical and 7-mm EVPAs after 29 October implies that the knot is the emitter of the polarized optical emission during the flare.

Previous authors ${ }^{14-16}$ have suggested that rotations of the polarization vector occur in BL Lac and the similar active galactic nucleus OJ 287. Their observations, which were more poorly sampled than ours, allowed multiple interpretations owing to the $\pm 180^{\circ}$ ambiguity of the EVPA. Despite this, the model that we advocate is quite similar to one of those proposed previously ${ }^{14,15}$, with the location of the emission region and connection with high-energy flares now specified by our sequences of Very Long Baseline Array images and multiwaveband light curves.

We interpret the event in the following manner (see Fig. 3). Explosive activity at the inlet of the jet near the black hole injects a surge of energy into the jet across part of its cross-sectional area. This disturbance appears as a knot of emission as it propagates along a subset of streamlines through the acceleration and collimation zone. Doppler beaming of radiation emitted by high-energy electrons in the disturbance increases as the knot accelerates along its spiral path, which stretches out with distance down the jet. These effects cause the flux of synchrotron radiation from the knot to rise until it dominates the optical, X-ray and (through inverse Compton scattering) $\gamma$-ray emission from BL Lac as the disturbance exits the zone of helical magnetic field. Maximum beaming - and therefore the peak in the light curve of the first flare-occurs during the last spiral, when the Lorentz factor of the jet is near its asymptotic value and the velocity vector of the knot points most closely towards our line of sight. The peak can be quite sharp ${ }^{5}$, as observed. At the point when the flare dominates the optical flux, we see the optical polarization vector rotate before the shock exits the acceleration and collimation zone. This zone is opaque at radio wavelengths, owing to synchrotron selfabsorption; hence, the first flare is absent in the radio light curves.

Beyond the acceleration and collimation zone, the disturbance forms a moving shock wave that encounters a region of turbulence, which is possibly driven by velocity shear across the jet ${ }^{6}$ downstream of the point at which the magnetic and particle energy densities reach rough equipartition ${ }^{4}$. The ambient magnetic field in the jet has a chaotic structure in this region. Because the shock front amplifies only the component of the field that is parallel to the front, the EVPA becomes transverse to this direction and therefore essentially

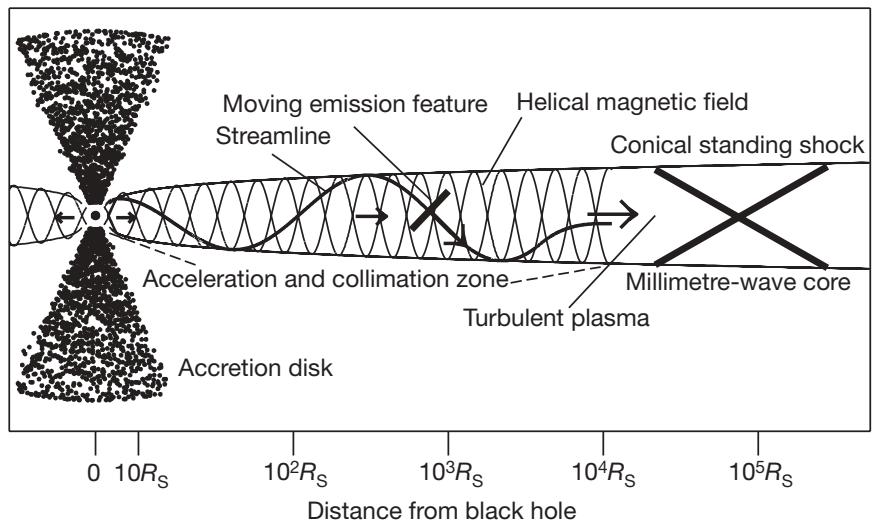

Figure 3 | Proposed model for the inner jet of BL Lac. A shock propagates down the jet along a spiral streamline. The first flare occurs during the last $240^{\circ}$ twist of the streamline before the flow straightens and becomes turbulent. The passage of the feature through the millimetre-wave core stimulates the second flare. A logarithmic scale of distance from the black hole, shown in terms of the Schwarzschild radius $\left(R_{\mathrm{S}}\right)$, is used to illustrate phenomena on various scales. parallel to the velocity vector of the knot at a position angle of $\sim 190^{\circ}$. During this phase, the flux lessens as the knot continues down the broadening jet, where the magnetic field strength and electron density decrease.

According to the model we propose here, the variation of EVPA with time should deviate from a strict linear dependence owing to projection effects, because the circular cross-section has an elliptical shape from our viewpoint. We have calculated this effect, including relativistic aberration, and show in Fig. $2 \mathrm{~g}$ that the optical EVPA data do follow the predicted curve. The small number of brief excursions of the EVPA from the curve, the deviations from the mean EVPA before and after the rotation, and irregularities in the light curves can all be explained by local flare-ups of emission that briefly amplify both the polarization along a particular direction and the flux at various wavebands.

The smoothness of the plot of EVPA versus time eliminates the possibility ${ }^{16,17}$ that the rotation is actually a random walk of the polarization vector due to a chaotic magnetic field. If this were the case, our numerical simulations (see ref. 17) indicate that the curve would be much more jagged than is observed when the degree of polarization is $\sim 5 \%$. In the simulations, this level of polarization corresponds to synchrotron emission from $\sim 200$ independent cells, each with a randomly oriented magnetic field. Apparent rotations by $\sim 240^{\circ}$ are very rare in such simulations, whereas they are common during flares of BL Lac and similar objects ${ }^{14}$.

Both synchrotron radiation and inverse Compton scattering contribute to the X-ray emission from BL Lac, with synchrotron radiation dominating when electrons are accelerated to energies in the $\mathrm{TeV}$ range $\mathrm{e}^{18,19}$. This generally causes the plot of flux density $\left(F_{v}\right)$ versus frequency $(v)$ to steepen in the X-ray range in such a way that the spectral index $\alpha$ is greater than one, where $F_{v} \propto v^{-\alpha}$. Such X-ray spectral steepening occurs during the first flare. In contrast, the X-ray spectrum becomes harder $(\alpha<1)$ during the second flare, as is expected if the X-rays are mostly generated by inverse Compton scattering of optical and infrared photons.

The second flare, which started at 2005.89, is simultaneous with the passage of the knot through the core seen on the Very Long Baseline Array images. If the core were a standing conical shock, as has been determined from simultaneous radio and optical polarization variability in the case of the quasar PKS 0420-014 (ref. 18), the emission would increase as the knot undergoes compression by the shock front. The flare dies down at optical and X-ray frequencies as the knot propagates away from the core down the expanding jet. However, it lasts much longer at $43 \mathrm{GHz}$, at which frequency the synchrotron radiation requires lower-energy electrons that have longer energetic lifetimes than those emitting at higher frequencies.

In the Supplementary Information we relate the angular velocity of the feature, inferred from the rotation of the optical polarization vector, to the rotational velocity of the base of the magnetic field near the black hole. We find that the rotational velocity thus obtained is consistent with the predictions of models in which the jet is driven by twisting magnetic fields from either the accretion $\operatorname{disk}^{1,3,4,5}$ or the ergosphere of the black hole $e^{2,3,20,21}$.

The combination of densely sampled sub-milliarcsecond imaging using the Very Long Baseline Array, polarimetry, and multiwaveband flux measurements has allowed us to explore the inner jet of BL Lac. Future data from more sensitive $\gamma$-ray Cherenkov detectors and the NASA Gamma-ray Large Area Space Telescope will soon allow more refined analyses. The ability to detect emission upstream of the core at 7-mm wavelength indicates that increasing the resolution of verylong-baseline interferometry by adding antennas in high Earth orbits will provide more detailed direct imaging of the inner jets of active galactic nuclei.

\section{Received 17 January; accepted 6 March 2008.}

1. Blandford, R. D. \& Payne, D. G. Hydromagnetic flows from accretion discs and the production of radio jets. Mon. Not. R. Astron. Soc. 199, 883-903 (1982). 
2. Blandford, R. D. \& Znajek, R. Electromagnetic extraction of energy from Kerr black holes. Mon. Not. R. Astron. Soc. 179, 433-456 (1977).

3. Meier, D. L., Koide, S. \& Uchida, Y. Magnetohydrodynamic production of relativistic jets. Science 291, 84-92 (2001).

4. Vlahakis, N. \& Königl, A. Magnetic driving of relativistic outflows in active galactic nuclei. I. Interpretation of parsec-scale accelerations. Astrophys. J. 605, 656-661 (2004).

5. Vlahakis, N. in Blazar Variability Workshop Il: Entering the GLAST Era (ASP Conf. Ser. 350) (eds Miller, H. R. Marshall, K., Webb, J. R. \& Aller, M. F.) 169-177 (Astronomical Society of the Pacific, San Francisco, 2006).

6. Jorstad, S. G. et al. Polarimetric observations of 15 active galactic nuclei at high frequencies: jet kinematics from bimonthly monitoring with the Very Long Baseline Array. Astron. J. 130, 1418-1466 (2005)

7. Albert, J. et al. Discovery of very high energy $\gamma$-ray emission from the lowfrequency peaked BL Lac object BL Lacertae. Astrophys. J. 666, L17-L20 (2007),

8. Jorstad, S. G. et al. Multi-epoch VLBA observations of EGRET-detected quasars and BL Lac objects: connection between superluminal ejections and gamma-ray flares in Blazars. Astrophys. J. 556, 738-748 (2001).

9. Lähteenmäki, A. \& Valtaoja, E. Testing of inverse Compton models for active galactic nuclei with gamma-ray and radio observations. Astrophys. J. 590, 95-108 (2003).

10. Junor, W. Biretta, J. A. \& Livio, M. Formation of the radio jet in M87 at 100 Schwarzschild radii from the central black hole. Nature 401, 891-892 (1999).

11. Melia, F., Liu, S. \& Fatuzzo, F. Is thermal expansion driving the initial gas ejection in NGC 6251? Astrophys. J. 567, 811-816 (2002).

12. Laing, R. A., Parma, P., de Ruiter, H. R. \& Fanti, R. Asymmetries in the jets of weak radio galaxies. Mon. Not. R. Astron. Soc. 306, 513-530 (1999).

13. Lyutikov, M., Pariev, V. I. \& Gabuzda, D. C. Polarisation and structure of relativistic parsec-scale AGN jets. Mon. Not. R. Astron. Soc. 360, 869-891 (2005).

14. Sillanpää, A., Takalo, L. O., Nilsson, K. \& Kikuchi, S. Photopolarimetry of BL Lac. Astrophys. Space Sci. 206, 55-70 (1993).
15. Moore, R. L. et al. The noise of BL Lacertae. Astrophys. J. 260, 415-436 (1982).

16. Kikuchi, S., Inoue, M., Mikami, Y., Tabara, H. \& Kato, T. A synchronous variation of polarization angle in OJ 287 in the optical and radio regions. Astron. Astrophys. 190, L8-L10 (1988).

17. D'Arcangelo, F. D. et al. Rapid multiwaveband polarization variability in the quasar PKS 0420-014: optical emission from the compact radio jet. Astrophys. J. 659, L107-L110 (2007)

18. Böttcher, M. et al. Coordinated multiwavelength observations of BL Lacertae in 2000. Astrophys. J. 596, 847-859 (2003).

19. Tanihata, C. et al. Rapid synchrotron flares from BL Lacertae detected by ASCA and RXTE. Astrophys. J. 543, 124-130 (2000)

20. McKinney, J. C. \& Narayan, R. Disk-jet coupling in black hole accretion systems II: force-free electrodynamical models. Mon. Not. R. Astron. Soc. 375, 531-547 (2007).

21. Hawley, J. \& Krolik, J. H. Magnetically driven jets in the Kerr metric. Astrophys. J. 641, 103-116 (2006).

22. Freedman, W. L. et al. Final results from the Hubble Space Telescope key project to measure the Hubble constant. Astrophys. J. 553, 47-72 (2001).

Supplementary Information is linked to the online version of the paper at www.nature.com/nature.

Acknowledgements The authors thank N. Vlahakis for discussions. The research reported here is based on work supported in part by the US National Science Foundation, NASA, the Russian Fund for Basic Research, the Academy of Finland, and the Italian Space Agency (ASI). The Very Long Baseline Array is an instrument of the National Radio Astronomy Observatory, a facility of the National Science Foundation operated under cooperative agreement by Associated Universities, Inc.

Author Information Reprints and permissions information is available at www.nature.com/reprints. Correspondence and requests for materials should be addressed to A.P.M. (marscher@bu.edu). 